\title{
Cyanide ingestion
}

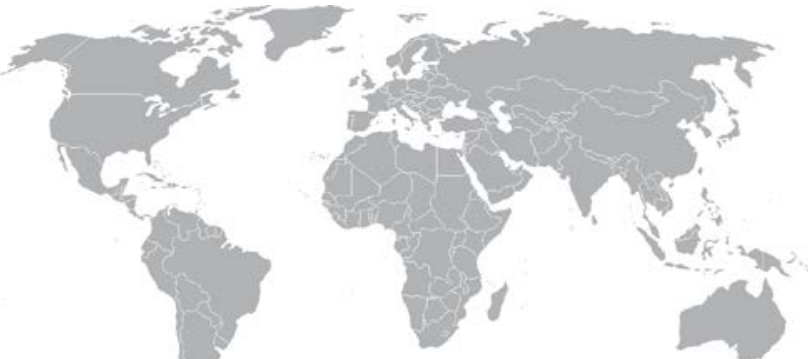

Abuzer Coskun ${ }^{1}$, Fikret Ozkan², Sedat Ozbay ${ }^{1}$, Osman Mahir Okur ${ }^{1}$, Afsin Emre Kayipmaz ${ }^{3}$, Behnan Gulunay ${ }^{1}$, Sevki Hakan ${ }^{2}$ Eren $^{4}$, Asuman Ucar ${ }^{1}$, Cemil Kavalci ${ }^{3}$

\section{ABST RACT}

Cyanide is one of the oldest poisons. It has recently been introduced into industrial use in the gold enrichment process in gold mines. A 36 -year-old man engaged in silver polishing business was brought to our emergency department by his colleagues with impaired consciousness and foamy saliva expectoration after accidentally drinking a sip of water mixed cyanide used for silver polishing. His general status was poor, and he was unconscious upon admission. The patient was administered the antidote three hours after his admission and extubated 11 hours after admission. His vital signs gradually returned to normal. On follow-up his urine color turned red; therefore, a second consultation was made with the poison information center, which recommended administering a second dose of antidote. After doing so, the patient gradually recovered, and he was discharged with normal liver enzymes, normal consciousness, and good overall status on 4th day of admission.

Keywords: cyanide, emergency, intoxication

\section{INTRODUCTION}

Cyanide is one of the oldest poisons. It has a bad reputation owing to being used as an agent used for military purposes. It was used in Nazi Germany, Peoples Temple sect mass suicide in Guyana, and the World Trade Center attacks in $2003(1,2)$. Cyanide has recently been introduced into industrial use in the gold enrichment process in gold mines. Cyanide can be industrially or naturally produced. The main sources of cyanide include plastic manufacturing, mining, photography, production of disinfectants and fertilizers, as a combustion residue of some materials (wool, nylon, silk, melamine, polyurethane etc), certain drugs (e.g. sodium nitroprusside), cigarette smoke and exhaust fumes, and fruits of some plants (apricot, cherry, plum, peach). Cyanide may be in solid, liquid, or gaseous states. While its gaseous and liquid states are colorless, the solid state is white in color. Cyanide intoxication may occur in acute or chronic forms. The intoxication occurs via absorption through skin, smoke inhalation, or more rarely, accidental oral intake $(1,2)$.

In this report, we discussed a rare case of cyanide intoxication by oral ingestion of cyanide from a glass with a review of the relevant literature.

\section{CASE REPORT}

A 36-year-old man engaged in silver polishing business was brought to our emergency department by his colleagues with impaired consciousness and foamy saliva expectoration after accidentally drinking a sip of water mixed cyanide used for silver polishing. His general status was poor, and he was unconscious upon admission. While his pupils were isochoric at the time of admission, he developed anisocoria 20-25 minutes later. His respiratory system examination revealed diminished breath sounds but no rales or rhonchi. Cardiovascular examination was notable for mild tachycardia but no additional heart sounds or murmur. On neurological examination no neurological deficit was detected. His vital signs included a blood pressure of $80 / 50 \mathrm{mmHg}$, pulse rate of 117 beats per minute, respiratory rate of 24 breaths per minute, and body temperature of $35.1^{\circ} \mathrm{C}$. The laboratory results were as follows: Hemoglobin: $17.3 \mathrm{~g} / \mathrm{dL}(13.5-18 \mathrm{~g} / \mathrm{dL})$, platelet count: $279000 / \mu \mathrm{L}$ (150000-400000/ $\mu \mathrm{L})$, prothrombine time: 10.1 seconds (11.5-14.5 seconds), INR: 0.96 (0.8-1.2), activated partial thromboplastin time (APTT): 24.6 seconds $(22-40$ seconds), lactate dehydrogenase (LDH): $341 \mathrm{U} / \mathrm{L}(210-425 \mathrm{U} / \mathrm{L})$, aspartate aminotransferase (AST): $37 \mathrm{U} / \mathrm{L}$ (5-34 U/L), alanine aminotransferase (ALT): $29 \mathrm{U} / \mathrm{L}(0-55 \mathrm{U} / \mathrm{L})$, C-reactive protein (CRP): $6.13 \mathrm{mg} / \mathrm{L}(0-5 \mathrm{mg} / \mathrm{L})$. His arterial blood gas analysis showed the following results: $\mathrm{pH}$ : 7.02 (7.35-7.45), partial oxygen pressure (PaO2): $78.8 \mathrm{mmHg}(80-100 \mathrm{mmHg})$, bicarbonate level (HCO3): $22.1 \mathrm{mEq} / \mathrm{L}(22-26 \mathrm{mEq} / \mathrm{L})$, partial carbon dioxide pressure (PaCO2): $34.1 \mathrm{mmHg}(35-45 \mathrm{mmHg})$, oxygen saturation level (SpO2): $97.9 \%(95-97 \%$ ). The patient was admitted to the emergency department with the preliminary diagnosis of cyanide intoxication. The case was consulted with the poison information center via telephone, and the patient was admitted to the intensive care unit. The patient first developed anisocoria at 1st hour after admission, followed by shallow breathing and bradycardia. A blood gas analysis indicated a pH level of 7.02 and the patient was thus intubated and connected to a mechanical ventilator. Since no antidote of cyanide was available in our hospital and within the borders of our province, a contact was made with 112 Emergency Ambulance Service to obtain the antidote, Hydroxicobalamine $5 \mathrm{~g}$ (Cyanokit, Pfizer Inc., New York, NY), from the nearby Kayseri Training and Research Hospital. The patient was administered the antidote three hours after his admission and extubated 11 hours after admission. His vital signs gradually returned to normal. On follow-up his urine color turned red; therefore, a second consultation was made with the poison information center, which recommended administering a second dose of antidote. After doing so, the patient gradually recovered, and he was discharged with normal liver enzymes, normal consciousness, and good overall status on 4th day of admission.

\footnotetext{
Numune Hospital, Department of Emergency, Sivas, Turkey

Numune Hospital, Department of Anaesthesiology and Reanimation, Intensive Care Unit, Sivas, Turkey

Baskent University Faculty of Medicine, Department of Emergency, Ankara, Turkey

Cumhuriyet University Faculty of Medicine, Department of Emergency, Sivas, Turkey

Received: 15 | uly 2015, Accepted: 12 Sep 2015
}

Correspondence: Afsin Emre Kayipmaz, MD

Baskent University Faculty of Medicine, Emergency Department, Bahcelievler, Ankara, Turkey Phone: +903122036868

Fax: +903122236439

E-mail: aekayipmaz@baskent.edu.tr 


\section{DISCUSSION}

Cyanide acts on the mitochondrial cytochrome oxidase system, thereby inhibiting oxidative phosphorylation and cellular respiration. Even when adequate oxygen is available within a medium, ATP production is reduced, and the aerobic mechanism is disrupted owing to impaired cytochrome oxidase system. As a result, hypoxia at cellular level takes place. Due to this mechanism of action, cyanide most profoundly affects tissues with the highest oxygen requirement. Hence, central nervous system and heart are the most severely affected tissues. The basic elimination mechanism of cyanide is via metabolization into thiocyanate by rhodanase in liver $(1,2,3)$.

Cyanide is a highly lethal poison. Many cases of fatality occurred after its entry into body after its accidental, suicidal, or homicidal use. Cyanide is found in many products in our daily life and encountering or obtaining cyanide quite simple. Persons having undergone long-term nitroprusside treatment may develop cyanide intoxication after intake of foods containing cyanide. Cyanide is also widely used in many industrial areas including plastic manufacturing, jewelry, and mining. Our patient was exposed to cyanide by inadvertently ingesting cyanide used for silver polishing. Cyanide may also release into air as a combustion product of some materials such as wool, nylon, silk, and acrylic. During fires, cyanide intoxication due to smoke inhalation may lead to fatalities independent of carbon monoxide $(1,2,3)$.

The clinical presentation of cyanide intoxication depends on the compound containing cyanide, the route of exposure, and the time since the exposure (1). In acute intoxications, clinical signs and symptoms emerge very rapidly. Hydrogen cyanide gas at doses less than 50 particles per million (ppm) causes restlessness, anxiety, palpitations, and difficulty breathing. At higher doses, cyanide causes impaired consciousness, seizures, and cardiac arrhythmias. At very high levels, hydrogen cyanide leads to cardiovascular collapse, coma, and death. The median lethal doses (LD50) for 30-minute and 5-minute exposures to hydrogen cyanide gas have been reported 200 ppm and 600-700 ppm, respectively. The action of solid cyanide salt begins within a few minutes after its intake. In a normal healthy adult, the lethal dose of cyanide salt has been reported to be 140 to 250 $\mathrm{mg}$, although doses as low as $50 \mathrm{mg}$ can also result in death (2, $3,4)$.

The diagnosis of cyanide intoxication is made by the clues obtained from patient history and clinical examination. As the signs and symptoms of cyanide intoxication begin too rapidly, every minute passing after intake is literally a matter of life and death. The treatment should immediately begin upon patient arrival under the light of goal-directed history and physical examination, without awaiting laboratory tests. Particularly exposures via inhalation are rapidly fatal, with signs and symptoms appearing within seconds and death within a couple of minutes; however, emergence of signs and symptoms may be delayed for up to 30 minutes with oral ingestion $(2,4,5,6)$.

\section{REFERENCES}

1. Gresham C, LoVecchio F. Industrial toxins. In: Tintinalli J E, Stapczynski SJ , Ma J O, Cline DM, Cydulka RK, Meckler GD, editors. Tintinalli's emergency medicine: a comprehensive study guide. 7th ed. New York, NY: The McGraw-Hill Companies. 2011;1317-20.

2. Vural N. Hydrocyanic acid. In: Vural N. Toxicology. Ankara, Turkey: Ankara University Faculty of Pharmacy Publications. 2005:421-26.

3. Renklidag T, Karaman AG. Cyanide intoxication. Sted. 2003; 12(9): 350-3.
Our patient's symptoms similarly began 20 minutes after oral ingestion. Metabolic acidosis with wide anion gap, a high lactate level, and a normal $\mathrm{PaO} 2$ level in arterial blood gas analysis should bring to mind cyanide intoxication (1). Our patient also had severe metabolic acidosis in arterial blood gas analysis. Similarly, bradycardia without accompanying cyanosis should make clinicians suspect cyanide toxicity $(1,2,4)$. In cyanide intoxication skin color may acquire a cherry red color as a result of high venous hemoglobin concentration (1). Bitter almond smell in breath may also aid in the diagnosis $(1,4)$. Severe cases of intoxication are characterized by impaired consciousness, hypotension, bradycardia, and tachypnea $(1,2)$. Our case also had a severely impaired consciousness, hypotension, and tachypnea.

In management of cyanide intoxication, it is vital to provide $100 \%$ oxygen and ventilatory support $(1,2,6)$. Hypotension can be treated with vasopressors and crystalloids. Acidosis should be treated with ventilatory support and sodium bicarbonate treatment (1). Our case also had severe acidemia for which he was intubated and connected to a mechanical ventilator. Specific antidote therapy should be commenced at once in cyanide intoxication $(1,2)$. Antidotes of cyanide include nitrites (amyl nitrite and sodium nitrite), sodium thiosulphate, hydroxycobalamine, dimethylaminophenol, dicobalt edetate, and hyperbaric oxygen $(1,2)$. Nitrites produce methemoglobinemia to make cyanide dissociate from cytochrome. In clinical practice, amyl nitrite ampoule is crushed, and the patient is made sniff it; this is followed by intravenous amyl nitrite administration $(1,4)$. The most important side effects of nitrites are hypotension and methemoglobinemia $(1,2)$. Due to these potential side effects, alternative antidotes have been investigated. As a result, hydroxycobalamine (vitamin B12) was licensed as a cyanide antidote in 1996 (1). Thanks to its low toxicity and high efficacy profile, its use is recommended in out-of-hospital settings and in cases when no clear diagnosis can be established $(1,7)$. Its potential side effects include transient hypertension and anaphylaxis (1). We also administered hydroxycobalamine (Cyanokit, Pfizer Inc., New York, NY) to our patient. Hyperbaric oxygen is recommended especially in the event of smoke inhalations in fires, when cyanide intoxication occurs together with carbon monoxide intoxication (2). Dicobalt edetate is a cobalt compound similar to cyanide. Despite being highly efficacious as an antidote, increased toxicity in the absence of cyanide in a medium limit its use when a specific diagnosis cannot be made $(1,2,8)$.

In conclusion, cyanide intoxication is not common in clinical practice despite a wide range of areas of cyanide use. As a highly lethal intoxication, a correct diagnosis and an adequate management strategy are of utmost importance. Specific antidotes should always be kept in mind, and every effort should be made to obtain and administer it as early as possible in-patient management.

4. Kocak S, Dundar ZD, Demirci S, Cander B, Dogan H. Cyanide intoxication: A case report. AKATOS. 2010; 1(1): 11-3.

5. Jethava D, Gupta P, Kothari S, Rijhwani P, Kumar A. Acute cyanide intoxication: A rare case of survival. Indian J Anaesth. 2014;58(3):312-14.

6. Hall AH, Rumack BH. Clinical toxicology of cyanide. Ann Emerg Med. 1986; 15(9): 1067-74.

7. Hall AH, Dart R, Bogdan G. Sodium thiosulfate or hydroxocobalamin for the empiric treatment of cyanide poisoning? Ann Emerg Med. 2007; 49(6):806-13. 
8. Lawson-Smith $\mathrm{P}$, Jansen EC, Hyldegaard O. Cyanide intoxication as part of smoke inhalation-a review on diagnosis and treatment from the emergency perspective. Scand I Trauma Resusc Emerg Med. 2011:19(1): 14.

$\diamond \diamond \diamond \diamond \diamond \diamond \diamond$

http:/ / www.ejgm. org 